\title{
Photo-polymerization, swelling and mechanical properties of cellulose fibre reinforced poly(ethylene glycol) hydrogels
}

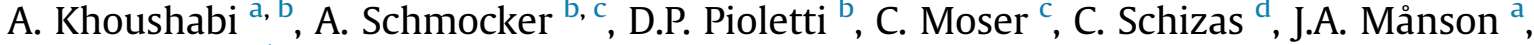 \\ P.E. Bourban ${ }^{a}$, * \\ a Ecole Polytechnique Fédérale de Lausanne (EPFL), Laboratory of Polymer and Composite Technology, Institute of Materials, Lausanne, Switzerland \\ ${ }^{\mathrm{b}}$ EPFL, Laboratory of Biomechanical Orthopedics, Institute of Bioengineering, Switzerland \\ ${ }^{c}$ EPFL, Laboratory of Applied Photonics Devices, Microengineering Institute, Switzerland \\ d Centre Hospitalier Universitaire Vaudois (CHUV), Orthopedic Department, Switzerland
}

\section{A R T I C L E I N F O}

\section{Article history:}

Received 9 July 2015

Received in revised form

1 October 2015

Accepted 4 October 2015

Available online 8 October 2015

\section{Keywords:}

Nano composites

Curing

Mechanical properties

Rheology

Hydrogel

\begin{abstract}
A B S T R A C T
The application of hydrogels as load-bearing biomedical components is often limited by their mechanical properties. Often an attempt to improve a hydrogel's stiffness is accompanied by a loss of toughness and swelling properties. In this work, we show that the addition of nanofibrillated cellulose (NFC) provides a mean to tailor both the swelling and the mechanical properties of the hydrogel. Various volume fractions of NFC were added to poly(ethylene glycol) dimethacrylate (PEGDM) precursors with two different molecular weights ( 6 and $20 \mathrm{kDa}$ ). The viscosity measurements of the precursor solutions indicated that the dispersed NFCs form a network-like structure in the hydrogel precursor. Such a structure, as observed in the photo-rheology experiments, serves as a light-scattering source when the solution is illuminated by UV light, which provides a uniform polymerization of the hydrogel in three-dimension and reduces the curing time. Mechanical properties of the neat and composite hydrogels were characterized using monotonic and cyclic compression tests. NFC reinforcement increases the hydrogel's stiffness by a factor 2 and 3.5 for the PEGDM matrixes with molecular weights of 6 and $20 \mathrm{kDa}$ respectively without compromising their toughness. Moreover, the desired stiffness and swelling properties can be simultaneously achieved by adapting the reinforcement concentration and the hydrogel cross-link density. The obtained composite hydrogels offer enhanced and tuneable properties and are proposed for injectable and photo-curable load-bearing implants.
\end{abstract}

๑ 2015 Elsevier Ltd. All rights reserved.

\section{Introduction}

Water swollen polymer networks, generally referred to as hydrogels, have received a great deal of attention for a wide variety of biomedical applications such as tissue engineering, cell encapsulation, wound dressing and drug delivery [1]. The hydrophilic polymer network of hydrogels allows a high water content similar to natural tissues. Their swelling properties, as well as their biocompatibility, makes hydrogels ideal candidate materials for the repair and regeneration of soft tissues.

Application of hydrogels as load-bearing components is often limited by their mechanical properties. Accordingly, a special emphasis is given in the literature to the synthesis of hydrogels

\footnotetext{
* Corresponding author.

E-mail address: pierre-etienne.bourban@epfl.ch (P.E. Bourban).
}

with enhanced mechanical performance [2-4]. It is well recognized that the stiffness of the polymer networks can be tailored with the cross-link density. Such an approach however results in an inverse proportionality between stiffness and toughness as described by the Lake-Thomas model [5].

Most methods developed to increase hydrogels' stiffness and toughness simultaneously require a chemical modification of the polymer and/or synthesis with several steps. This complexity in hydrogel synthesis is a drawback in cases where in situ formation of the hydrogel is required, such as injectable implants and cell seeding for cell encapsulations applications.

Designing a hybrid hydrogel is an effective approach to address the aforementioned issue while keeping a simple and fast processing routes. Yet, such a design clearly involves the choice of material components, their scale and shape. On this matter, the concept of composite hydrogels reinforced with an interpenetrated phase is introduced as a promising solution [6-9]. 
Cellulose nanofibre mats offer a great potential as reinforcement phase in hydrogel composites due to their large surface-to-volume ratio, high stiffness and strength. Introduction of a cellulose nanofibre in a covalently cross-linked polymer network can create physical entanglements of the cellulose fibres and the polymeric chains. Hence, the hydrophilicity of the cellulose fibres helps to keep the hydrogel's high equilibrium water content in the resulted composite. The integration of the cellulose fibres in the hydrogel is achieved either through the irreversible physical interactions or functionalization of the cellulose fibres enabling covalent crosslinking to the polymeric network.

Although nanofibre cellulose as reinforcement in polymer matrix composites was investigated $[10,11]$, the use of nanofibre cellulose in hydrogels [12] and the impact of nanofibre cellulose on the processing of hydrogels received few attention [13,14]. A detailed study linking material properties and material processing for such hybrid materials is still lacking.

In this work, the influence of nanofibrillated cellulose (NFC) addition to the photopolymerizable poly(ethylene glycol) dimethacrylate (PEGDM) hydrogel precursors of two different molecular weights is investigated. PEGDM was chosen as a matrix of the composite hydrogel due to its biocompatibility, tuneable properties and rapid photo curing [15]. Characterization of NFC addition to such a photopolymerizable hydrogel precursor substantially requires the assessment of the following aspects:

\subsection{Processability of the precursor solution}

An increase in the concentration of reinforcement in the polymeric precursor results in a change in the viscosity of the solution. The latter, as a key processing parameter affects the dispersion of the ingredients and consequently the homogeneity of the polymerized hydrogel. Furthermore, potential biomedical applications, such as the replacement of intervertebral disc tissue, often involve injection of the precursor and therefore require a low viscosity of the solution.

\subsection{Kinetics of photo-polymerization}

The interaction of the precursor solution and the incident radiation can be altered by the presence of fillers. The additional filler-phase scatters and absorbs light and also changes diffusion kinetics of the reacting species.

\subsection{Equilibrium water content and swelling capability}

The precise control of the swelling behaviour and water retention ability is of essential importance in design of hydrogel-based products. Any attempt to raise the concentration of the reinforcements has to take into account the associated effects on the consequent water uptake properties.

\subsection{Enhancement of the mechanical properties}

Ideally, the introduction of the reinforcement to the polymer network is a tool to tailor the hydrogel's mechanical properties according to the design requirements without any complex chemical procedures.

In the present work, the aforementioned aspects are investigated using a composite PEGDM hydrogels with different cross-link densities reinforced with NFC at different volume fractions. The viscosity of the precursor and photopolymerization kinetics were studied. The hydrogels equilibrium water content and volumetric swelling ratio were evaluated and the static and dynamic mechanical properties of the hydrogels under compression loading were investigated.

\section{Materials and methods}

\subsection{Materials synthesis}

\subsubsection{Poly(ethylene glycol) dimethacrylate (PEGDM)}

PEGDM was synthesized as previously described [16]. In summary, $20 \mathrm{~g}$ of dried poly(ethylene glycol) ( $\mathrm{Mw}=6$ and $20 \mathrm{kDa}$, Sigma Aldrich) were dissolved in dichloromethane (99.8\%, Extra Dry, Acros) and reacted with 3 molar equv. of methacrylic anhydride (94\%, Sigma Aldrich) and triethanolamine (99\%, Sigma Aldrich) under dry argon flow over five days. The solution was then precipitated in diethyl ether (99.5\%, Extra Dry, Acros), filtered and dried overnight.

\subsubsection{Nanofibrillated cellulose (NFC)}

The fibrillation was performed on cellulose pulp (bleached softwood pulp, Elemental Chlorine Free (ECF), Zellstoff Stendal, Germany) with a high-shear homogenizer, by pumping the suspension through two consecutive chambers of 400 and $200 \mu \mathrm{m}$ for 12 passes. As reported [17], the hemicellulose and cellulose contents are of 12.6 and $81.3 \%$ respectively while the diameters of NFC are in the range of $2-100 \mathrm{~nm}$ and their lengths are in a few micrometers range as observed by SEM.

\subsubsection{Hydrogels synthesis}

Hydrogel precursors were prepared by mixing PEGDM (10 wt.\%) with the molecular weights of 6 or $20 \mathrm{kDa}$, NFC, phosphate buffered saline (PBS, pH 7.4, Gibco) and 0.1 g.ml ${ }^{-1} \%$ of Irgacure 2959 (BASF), as photoinitiator. The two hydrogel matrices studied here are named PEGDM 6 kDa and PEGDM $20 \mathrm{kDa}$ according to their molecular weights. NFC was added to the hydrogel precursor with different volume fractions of $0.1,0.3,0.5,0.7$ vol.\%. Considering the density of the NFC the volume fractions were calculated from weight fractions.

Hydrogel precursors were homogenized using ultra-turrax (IKA T25 digital, SN 25 10G) and degassed at 30 mbar. They were then immediately cast in plastic moulds, covered with a microscope slide and irradiated during 30 min using a monochromatic $365 \mathrm{~nm}$ ultra violet lamp (AxonLab) with an intensity of $5 \mathrm{~mW} \cdot \mathrm{cm}^{-2}$. During illumination the radical polymerization of methacrylate groups of PEGDM molecule occurs. As a result, the PEGDM molecules are cross-linked and the three-dimensional structure of the hydrogel is built [18].

\subsection{Characterization}

\subsubsection{Precursor's viscosity}

The rheological behaviour of hydrogel precursors was evaluated using a rheometer (TA Instruments AR2000ex) in a parallel disc configuration with the disc diameter of $25 \mathrm{~mm}$. A precursors sample of $200 \mu \mathrm{l}$ was tested just after the mixing step. The precursor's viscosity evolution in strain sweep was recorded in oscillatory mode at $0.5 \mathrm{~Hz}$. The experimental error is calculated within the three replicates at the strain of $0.1 \%$.

\subsubsection{Photopolymerization kinetics}

The in situ measurement was carried on with the rheometer (same as Section 2.2.1) coupled with a custom built LED set-up with the wavelength of $365 \mathrm{~nm}$. The rheometer's lower quartz disc allowed the illumination of the sample during the measurement. The strain oscillation of $5 \%$ at a frequency of $10 \mathrm{~Hz}$ was applied. The evolution of the precursor's storage modulus $\mathrm{G}^{\prime}$ was measured during UV irradiation. The time required to reach $95 \%$ of the plateau level of $G^{\prime}$, called $t_{95}$, is reported.

In addition to all precursor formulations described in Section 
2.1.3, a precursor of PEGDM 6 kDa with 10 vol.\% Intralipids (Fresenius Kabi) was prepared to study the effect of the light scattering particle on photo-curing kinetics. The experimental error is calculated at the plateau level of $G^{\prime}$ within three replicates.

\subsubsection{Gel fraction}

Directly after synthesis, hydrogels were dried in a vacuum oven at $40{ }^{\circ} \mathrm{C}$ during $48 \mathrm{~h}$ and their weight $\left(\mathrm{W}_{\mathrm{d} 1}\right)$ was measured. They were then immersed in PBS and daily washed in order to remove the unreacted precursor. After 7 days, hydrogels were dried and their dried weight was measured for a second time $\left(\mathrm{W}_{\mathrm{d} 2}\right)$. The hydrogels' gel fraction (GF) was then calculated: $\mathrm{GF}$ (wt./wt.\%) $=W_{d 2} / W_{d 1} \times 100$.

In addition to all precursor formulations described in Section 2.1.3, the gel fraction of a set of PEGDM 6 kDa hydrogels with $50 \%$ lower concentration of photoinitiator (0.05 g.ml $\left.{ }^{-1 \%}\right)$ was investigated.

\subsubsection{Volumetric swelling ratio and water content}

Hydrogels were weighted after synthesis $\left(W_{0}\right)$, after 7 days of swelling in PBS $\left(\mathrm{W}_{\mathrm{s}}\right)$ and after drying $\left(\mathrm{W}_{\mathrm{d}}\right)$, using a density determination kit. Their corresponding volumes were then calculated based on Archimedes' buoyancy principles. The volumetric swelling ratio (SR) at equilibrium was determined via:

$S R(\%)=\left(V_{S}-V_{0}\right) / V_{0} \times 100$.

where $V_{0}$ is the volume of the samples after synthesis, $V_{s}$ is the volume of the swollen samples. The equilibrium water content (EWC) of hydrogels was measured by:

$E W C(\%)=\left(W_{s}-W_{d 2}\right) / W_{s} \times 100$.

\subsubsection{Mechanical testing}

Cylindrical hydrogels with the diameter of $8 \mathrm{~mm}$ and height of $4.5 \mathrm{~mm}$ were prepared for mechanical testing. The mechanical properties of the hydrogels in unconfined static and dynamic compression were evaluated using an Instron E3000 linear mechanical testing machine (Norwood, MA, USA) equipped with a $250 \mathrm{~N}$ load cell. During the test, swollen hydrogels were immersed in PBS. Hydrogels were compressed to $35 \%$ strain $\left(1 \mathrm{~mm} \cdot \mathrm{min}^{-1}\right)$, followed by 20 sinusoidal cycles of compression from $15 \%$ to $25 \%$ of strain at $1 \mathrm{~Hz}$. At the end samples were compressed at $80 \%$ strain. The elastic moduli (E) is calculated by linear interpolation of the true stress-strain curve between the strain of $0.15-0.25$. The modulus is measured a second time after 20 cycles of compression, the modulus variation is reported as: $E_{\text {after }} 20$ cycles/ $E_{\text {before applied cycles. }}$.

The failure stress and strain are respectively the maximum stress and strain that hydrogel tolerated in compression before breaking. Energy dissipation per unit of hydrogel volume is calculated as the averaged surface area of true stress-strain curve hysteresis over 20 loading-unloading cycles.

All values reported are a result of independent measurement of at least five replicates.

\section{Results and discussion}

\subsection{Hydrogel precursor viscosity}

In the fibre reinforced composite hydrogel, elevated viscosity of the precursor disturbs fibre dispersion in the matrix, precursor degassing procedure and moulding. It was observed that hydrogels with NFC concentrations higher than 0.7 vol.\% of PEGDM 20 and higher than 0.9 vol.\% of $6 \mathrm{kDa}$ are too viscous for homogeneous proper fibre dispersion and casting.

The viscosity evolution of PEGDM hydrogel precursors versus strain sweep is shown in Fig. 1. At the same NFC concentration and strain level, PEGDM $20 \mathrm{kDa}$ precursors have a higher viscosity compared to $6 \mathrm{kDa}$. This was expected due to the higher molecular weight of $20 \mathrm{kDa}$ than $6 \mathrm{kDa}$.

The NFC addition increases the precursor viscosity over the whole range of applied strains. This effect is more pronounced at low strain ranges, but still present at higher strain ranges (see insertion in Fig. 1(a)).

The viscosity of precursors containing NFC drastically decreases at higher strain levels. Since, no photo-curing is taking place during the viscosity measurement, this shear thickening behaviour is only the indication of a change between NFC-NFC or NFC-matrix interactions. Such an observation suggests that beyond a critical concentration of the NFC, network-like structure of the NFCs is formed through the formation of physical entanglements as described by Lowys et al. [19]. This continuous network can sustain relatively small strain levels, consequently providing an elevated viscosity. At higher strains (10-100\%) the precursor viscosity drops as a result of NFC network weakening or collapsing. At strain levels higher than $100 \%$ the network is completely broken and the higher viscosity of composite precursors compared to the neat ones is only due to the presence of the small fraction of NFC in the suspension.

\subsection{Hydrogel curing kinetics}

The evolution of shear storage modulus $\left(\mathrm{G}^{\prime}\right)$ as a function of UV illumination time is shown in Fig. 2(a) and (b). For all hydrogels, $G^{\prime}$ is initially low and constant in the radical polymerization initiation phase. The propagation phase starts by the reaction of methacrylate groups present at each PEGDM forming the poly(methacrylate). This cross-linking reaction transforms a liquid precursor into an elastic network. As a result, $\mathrm{G}^{\prime}$ rapidly increases while cross-linking continues. The increase in $G^{\prime}$ value slows down as the polymerization terminates and the curing finalizes.

The plateau level of $\mathrm{G}^{\prime}$ and $\mathrm{t}$ 95, are shown in Table 1. PEGDM $6 \mathrm{kDa}$ hydrogels have a shorter curing time and higher plateau level $\mathrm{G}^{\prime}$ than the $20 \mathrm{kDa}$ PEGDM hydrogels due to the presence of a higher concentration of cross-linkable methacrylate groups per volume in the $6 \mathrm{kDa}$ than in the $20 \mathrm{kDa}$ hydrogels.

In both hydrogels ( 6 and $20 \mathrm{kDa}$ ), the addition of NFC increases the hydrogel plateau level $\mathrm{G}^{\prime}$ and decreases the $t_{95}$. The acceleration of curing in the presence of the NFC could be due to the light scattering caused by the fibres networks which enhance the optical scattering coefficient of the precursor resulting in a higher total absorption coefficient per unit length.

The shorter curing time is of interest for practical applications. Specifically in biomedical or dental applications, for which a lower UV exposure time of cells or tissue and shorter surgery time are desirable. As we previously discussed [20], addition of light scattering particles, such as intralipids, is an effective method to decrease the required hydrogel curing time and achieve a photopolymerization of the volume. However, this curing acceleration is associated with decreasing the mechanical properties of the final material. In the case of a PEGDM $6 \mathrm{kDa}$ hydrogel, an addition of 10 vol.\% intralipids decreases the curing time by $26 \%$ and the plateau value $G^{\prime}$ value by $14 \%$. However, the addition of 0.7 vol.\% NFC to the improves both curing time and shear modulus by $36 \%$ and $110 \%$ respectively, hence offering a double advantage.

\subsection{Gel fraction}

The gel fraction indicates the amount of non-soluble cured network that is stable in a hydrogel three-dimensional structure. 

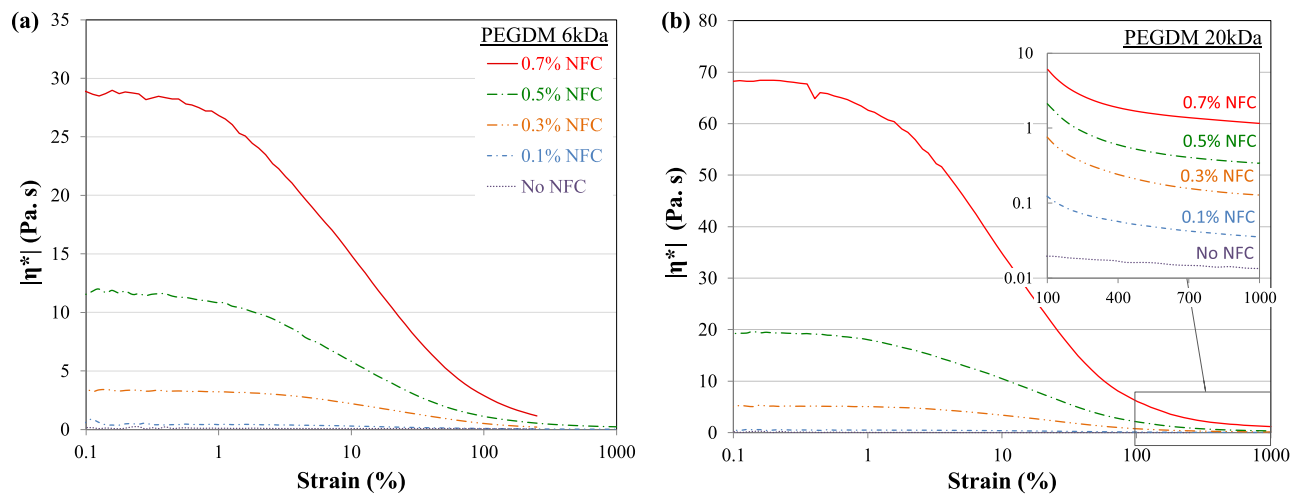

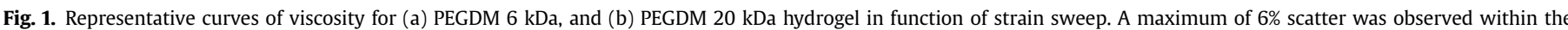
replicates of each formulation.
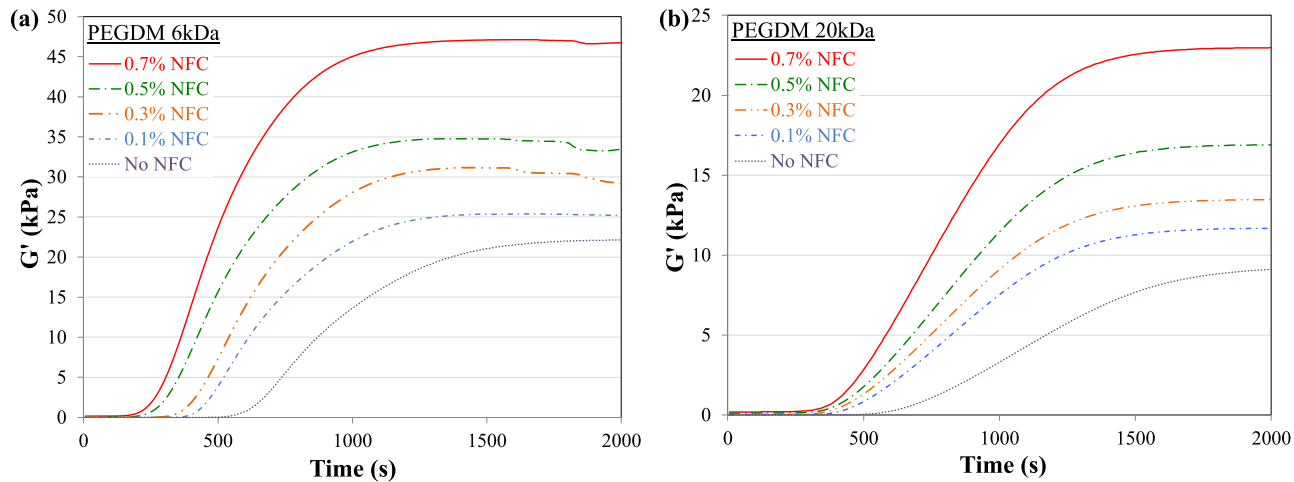

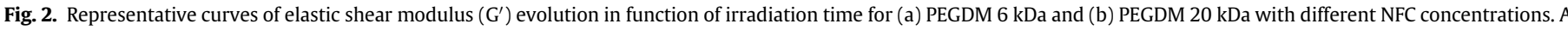
maximum of $4 \%$ scatter in $G^{\prime}$ values was observed within the replicates of each formulation.

Table 1

The plateau level $\mathrm{G}^{\prime}$ and the required time to achieve $95 \%$ of it ( $\left.\mathrm{t}_{95}\right)$ measured from the 6 and $20 \mathrm{kDa}$ PEGDM hydrogels.

\begin{tabular}{llrrrrlrrrr}
\hline Hydrogel & \multicolumn{3}{l}{ PEGDM 6 kDa } & \multicolumn{1}{c}{ PEGDM 20 kDa } \\
\hline NFC (vol.\%) & 0 & 0.1 & 0.3 & 0.5 & 0.7 & 0 & 0.1 & 0.3 & 0.5 & 0.7 \\
$\mathrm{G}^{\prime}$ (kPa) & 22 & 25.3 & 31.4 & 34.8 & 46.3 & 8.8 & 11.5 & 13.3 & 16.7 & 22.9 \\
$\mathrm{t}_{95}$ (min) & 24.6 & 19.1 & 18.6 & 16.4 & 15.6 & 27.3 & 23.1 & 22.8 & 22.9 & 22.1 \\
\hline
\end{tabular}

The unreacted precursor and the reinforcements that are not linked to the elastomeric network are washed off and the remaining fraction of the gel is reported as a gel fraction.

Fig. 3 illustrates the gel fraction of hydrogels synthesized with two different photoinitiator concentrations. The gel fraction of the hydrogels with 0.01 g.ml $\mathrm{ml}^{-1}$ photoinitiator are always higher than $94 \%$ independent of the hydrogels' molecular weight and fibre concentration.

Obtaining the same polymerization degree while reducing the photoinitiator amount is of particular interest in biomedical applications as it reduces the potential toxicity of the hydrogels precursor by minimizing the probability of photoinitiator's radicals reaction with nearby cells and tissue [21]. As suggested in Section 3.2 the NFC can be employed to enhance the polymerization kinetics which would allow to reduce the photo initiator concentration. This was confirmed by an evaluation of the gel fraction of a 6 kDa PEGDM hydrogel with 0.05 g.ml ${ }^{-1} \%$ concentration of photoinitiator (Fig. 3 dashed line). By using only half the photoinitiator concentration, the neat hydrogel gel fraction decreases from $94 \%$ to $60 \%$. However, when adding the NFC this decrease in photoinitiator

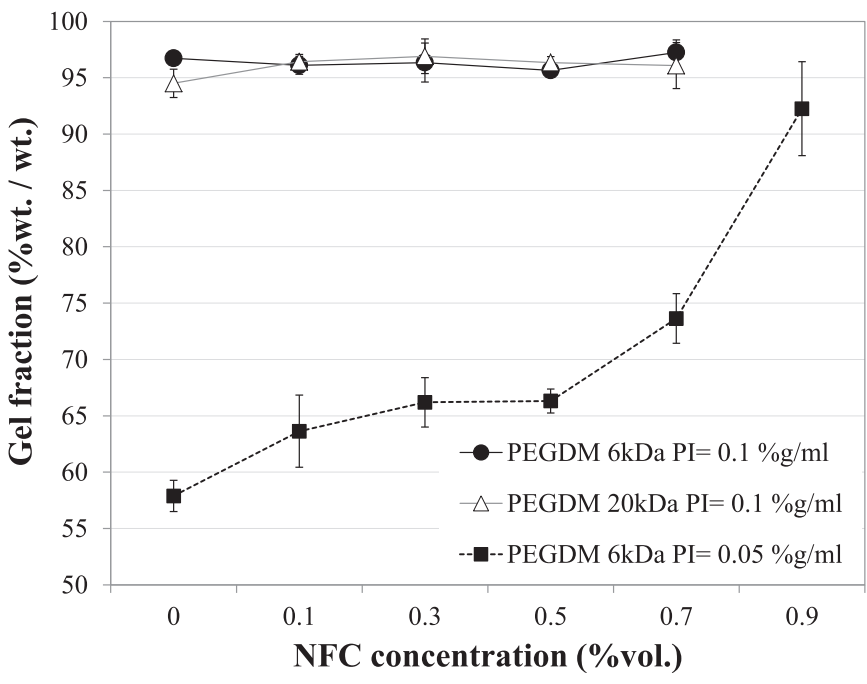

Fig. 3. Gel fraction of hydrogels synthesised with the regular photo initiator concentrate of $0.1 \mathrm{~g} / \mathrm{ml} \%$ (solid lines) and $0.05 \mathrm{~g} / \mathrm{ml} \%$ of photo initiator (dashed-line).

is compensated and at 0.9 vol.\% NFC a gel fraction of $92 \%$ is achieved.

\subsection{Equilibrium water content (EWC) and swelling ratio}

The EWC of the hydrogels are shown in Fig. 4. As expected, the 20 kDa PEGDM neat hydrogel with lower cross-link density has a 


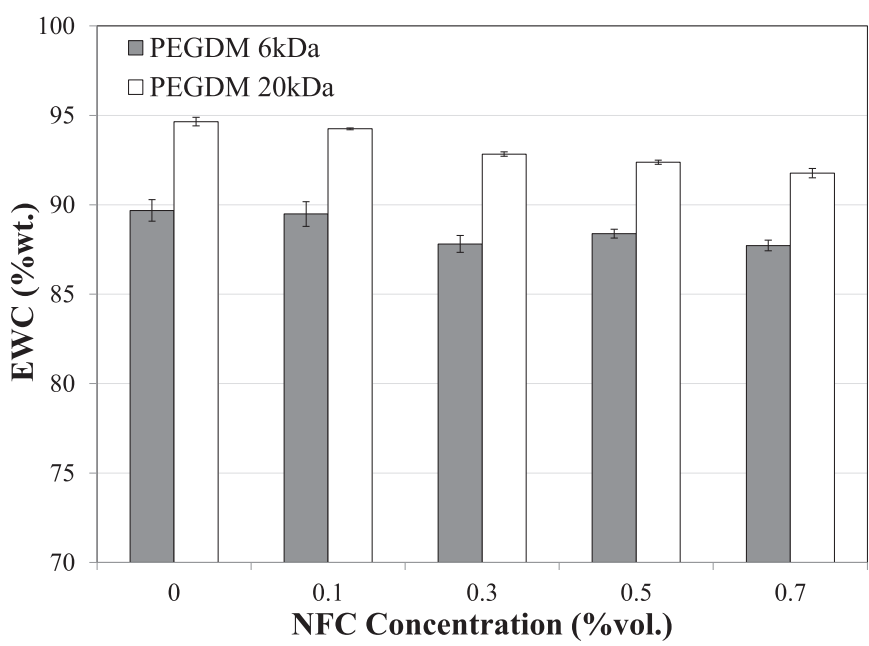

Fig. 4. The equilibrium water content (EWC) of the hydrogels.

higher EWC than the $6 \mathrm{kDa}$. A slight reduction in EWC is observed when adding NFC, e.g. EWC is decreased from 95 to $92 \mathrm{wt} . \%$ for $20 \mathrm{kDa}$ and from 90 to $88 \mathrm{wt}$.\% for $6 \mathrm{kDa}$. The NFCs entanglements with each other and with the polymeric network act as physical cross-link site which results in an increase of the total cross-link density of the network. This slightly decreases the effective mesh size of the hydrogel and decreases the EWC as a result. Note that, the initial water content of all the synthesized hydrogels is $90 \mathrm{wt} . \%$ and the lower EWC observed in $6 \mathrm{kDa}$ hydrogels is reached due to the network shrinkage.

Fig. 5 illustrates the volumetric swelling ratio of the hydrogels. The neat $20 \mathrm{kDa}$ hydrogel swells by 100 vol.\% of its initial volume whereas, the $6 \mathrm{kDa}$ hydrogel by $10 \mathrm{vol} \%$. The addition of NFC decreases the swelling ratio for both hydrogels. While a $20 \mathrm{kDa}$ PEGDM reinforced with NFC swells by a minimum of $50 \mathrm{vol} . \%$, a slight shrinkage is observed in case of the $6 \mathrm{kDa}$ composite hydrogels. This implies that the swelling ratio of the composite hydrogels can be effectively tuned by alternating the NFC concentration while the high EWC is preserved.

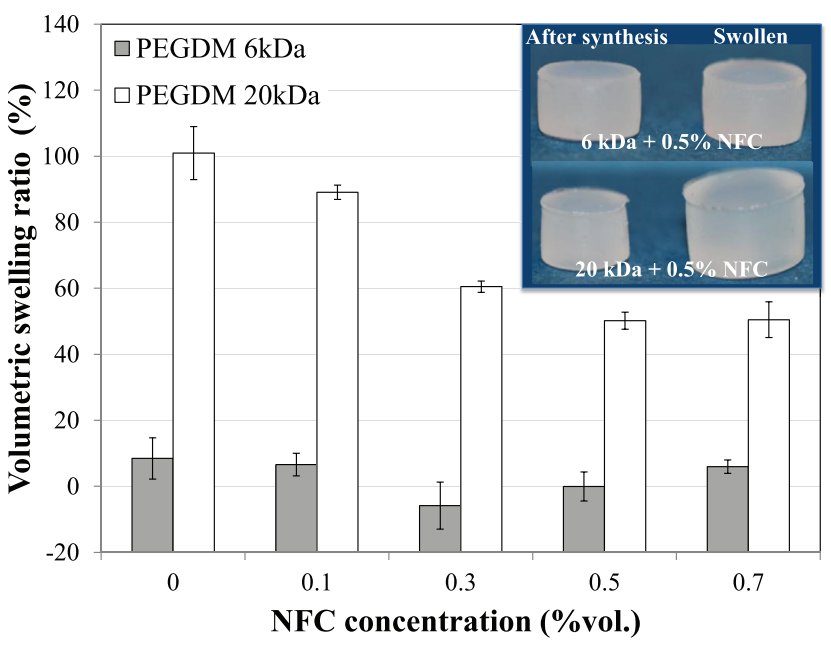

Fig. 5. Volumetric swelling ratio of hydrogels. The image shows the 6 and $20 \mathrm{kDa}$ PEGDM hydrogel reinforced with 0.5 vol.\% NFC just after synthesis and in swollen state.

\subsection{Hydrogels mechanical properties}

In Fig. 6 the elastic modulus of hydrogels is presented. The $6 \mathrm{kDa}$ hydrogel with higher cross-link density has a higher compression modulus than the $20 \mathrm{kDa}$ hydrogel. A significant increase of the hydrogels' moduli is achieved by NFC addition, e.g. addition of 0.7 vol.\% NFC increases the modulus by a factor 2 for $6 \mathrm{kDa}$ and a factor 3.5 for the $20 \mathrm{kDa}$.

This increase in hydrogel stiffness via the addition of NFC is a simple method that does not require an elaborated chemical modification of the hydrogel and is not associated with water lost, e.g. the addition of $0.5 \mathrm{vol}$ \% NFC to the neat PEGDM $20 \mathrm{kDa}$ matrix, increases the modulus to the level of neat PEGDM $6 \mathrm{kDa}$ while keeping a high EWC (92\%) and swelling ratio (50\%) of the hydrogel.

No failure was observed in the $20 \mathrm{kDa}$ hydrogels at the maximum applied compression of $80 \%$. Fig. 7 shows the true failure stresses and corresponding engineering failure strains of the $6 \mathrm{kDa}$ hydrogels. Neat hydrogel failed at $63 \%$ compression at an ultimate stress of $200 \mathrm{kPa}$. The failure strain is increases by $12 \%$ when adding 0.7 vol.\% NFC. The ultimate stress significantly increased (180\%) by addition of 0.7 vol.\% NFC.

The results of monotonic mechanical testing presented in Fig. 6

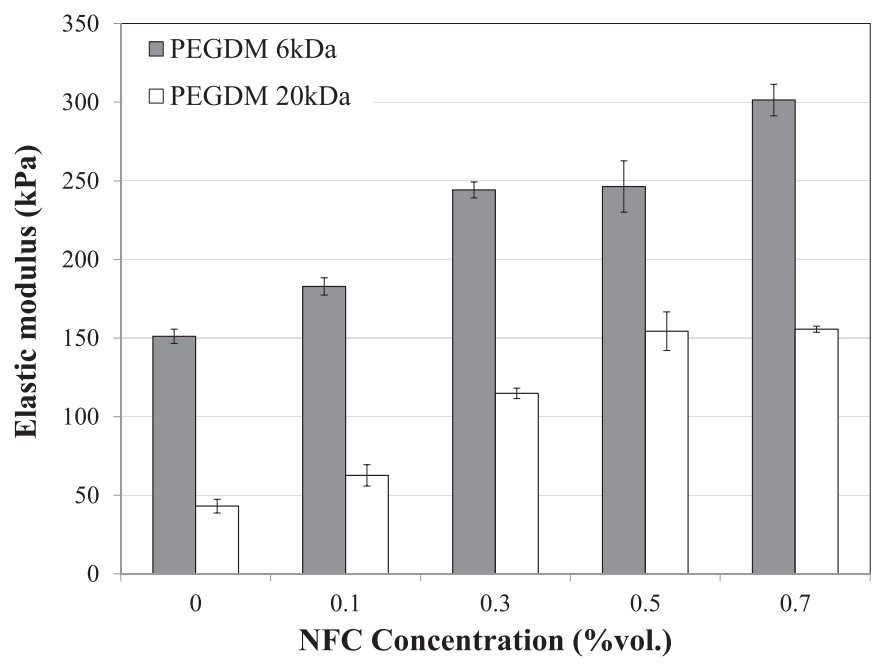

Fig. 6. Elastic moduli of the hydrogels.

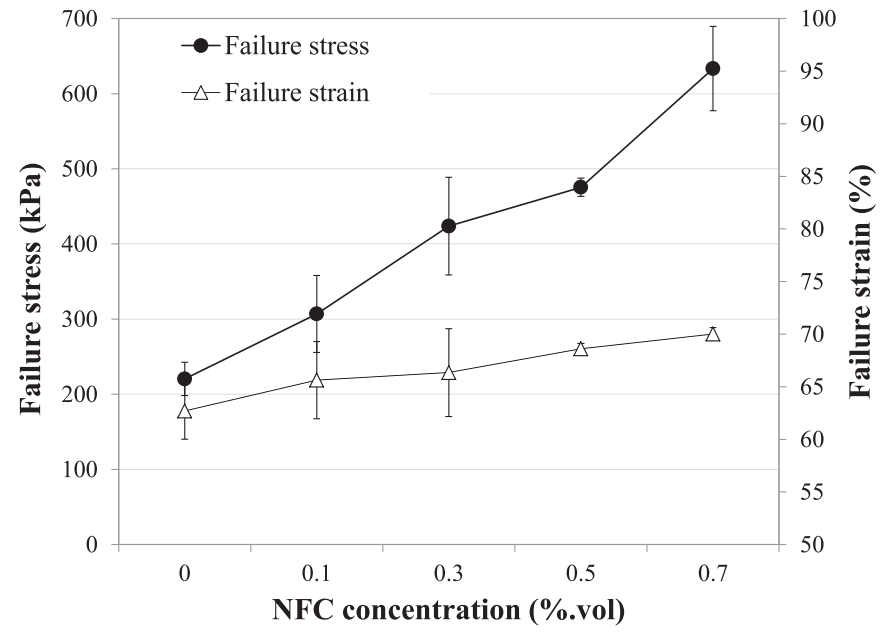

Fig. 7. The failure stress (solid line) and failure strain (dashed line) of PEGDM 6 kDa with different volume fraction of the NFC. 
and Fig. 7 suggest that the addition of fibre reinforcement is an effective method to improve stiffness and strength simultaneously. Fig. 8 illustrates the change in the elastic modulus of the hydrogels after 20 cyclic deformations.

The results indicate almost no stiffness variation of the neat hydrogels after the cyclic deformation, while a modulus reduction of up to $15 \%$ is observed in the case of the composite hydrogels. This stiffness reduction becomes more pronounced by increasing the NFC volume fraction. The later observation, as indicated in Fig. 8 is almost independent of the PEGDM matrix cross-link density. Therefore, the stiffness reduction in the composite hydrogels after cyclic deformation can be attributed to a certain degradation of the integrity in the NFC network structure discussed in Section 3.1.

The energy dissipation data in cyclic loading of the hydrogels are indicated in Fig. 9. The energy dissipation of the $6 \mathrm{kDa}$ is higher than the $20 \mathrm{kDa}$ neat hydrogel owing to the viscoelastic behaviours induced by the difference in cross-link densities. This is the main dissipative phenomenon as there is no significant variation in moduli before and after cyclic loading which is an indication of the absence of damage or cracks dissipating energy.

The addition of NFC increases the dissipated energy. The latter can be attributed to the energy dissipated by degradation of the NFC integrity as discussed before. However, the latter is not the only reason for the higher energy dissipation in the composites. Indeed, the modulus reduction is same for the $6 \mathrm{kDa}$ hydrogel reinforced with 0.5 and 0.7 vol.\% NFC (Fig. 8). However the dissipated energy is higher for the 0.7 vol.\% composite (Fig. 9).

Phenomena like physical interactions at the interfaces between NFCs or between fibres and the hydrogel matrix as well as the viscoelastic deformation of the NFC network might dissipate more energy in a composite with higher fibres content.

\section{Conclusion}

This study suggests that the addition of nanofibrillated cellulose (NFC) to the precursor solution of poly(ethylene glycol) dimethacrylate (PEGDM) hydrogels is an effective approach to tailor photopolymerization kinetics and mechanical properties of the resulting composite hydrogels. The rheology results show that dispersed NFCs form a network-like structure in the PEGDM solution. Such a structure serves as an effective light-scattering media when the precursor solution is illuminated by UV radiation and consequently reduces the curing time by $36 \%$ when adding 0.7 vol.\%

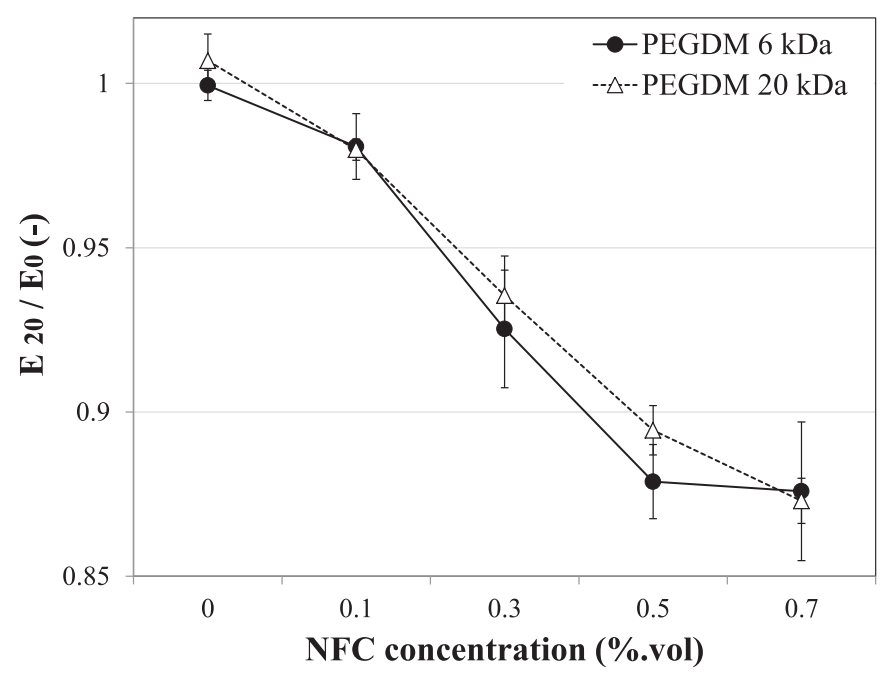

Fig. 8. Modulus variation of the neat and composite hydrogels after 20 loading cycle.

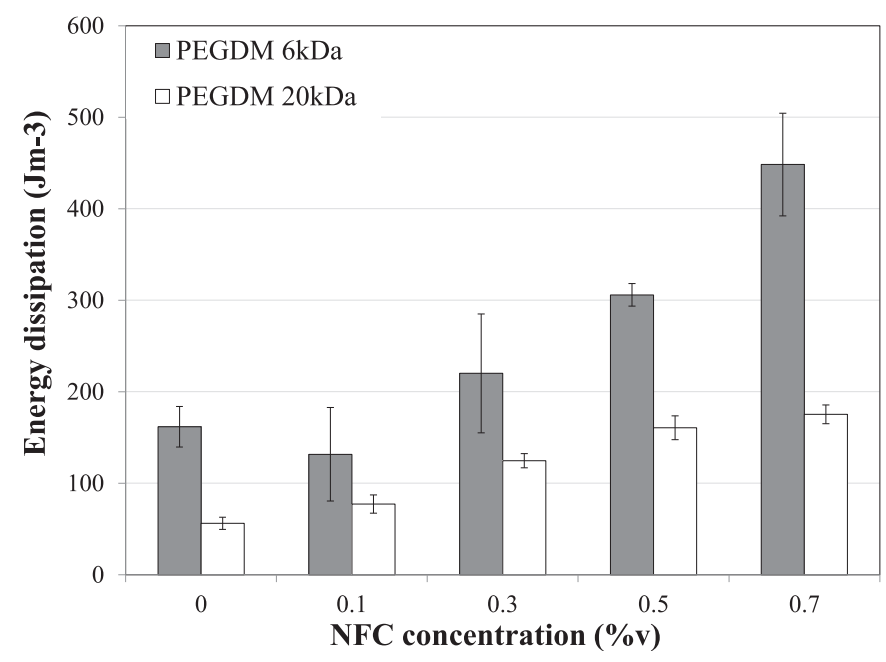

Fig. 9. Energy dissipation of hydrogels measured over 20 compression cycles.

of fibres. It is shown as well that the required concentration of photoinitiator can be reduced due to the accelerated kinetics of photo initiation in presence of NFC. Furthermore the influence of NFC on the swelling behaviour of these composite hydrogels having more than $88 \%$ of water, depends on the hydrogel matrix cross-link density. For example, the addition of 0.5 vol.\% NFC decreases the swelling ratio of the high molecular weight hydrogel matrix from 100 to 50 vol.\% while a slight shrinkage is observed in case of the lower molecular weight matrix. The results indicate that the NFC reinforcement improves the hydrogels stiffness without compromising its ultimate stress and strain. For example, the addition of 0.7 vol.\% NFC increases the modulus from 150 to $300 \mathrm{kPa}$ for a PEGDM $6 \mathrm{kDa}$ hydrogel and from 40 to $150 \mathrm{kPa}$ in the case of $20 \mathrm{kDa}$. At the same time this fibre addition increases the failure stress (from 220 to $630 \mathrm{kPa}$ ) and strain (from 62\% to 70\%) in the case of the $6 \mathrm{kDa}$ PEGDM hydrogel.

The enhanced and tuneable mechanical properties of the PEGDM-NFC composite hydrogels as an injectable and in-situ curable material make them promising candidates for loadbearing biomedical applications. This work also opens up a new pathway to increase the structural performance of biomaterials while not decreasing their biological performance such as the high water content required for cell seeding.

\section{Acknowledgement}

This work was financed by the Swiss National Science Foundation (grant CR 2312-137743). The Applied Wood Materials Laboratory, led by Dr T. Zimmerman at EMPA Dübendorf, Switzerland, provided the NFCs. In particular Dr Ph. Tingaut, Dr S. Josset and Dr H. Sehaqui are acknowledged for their support in preparing the NFCs. We thank the group of Prof. H. Frauenrath at EPFL, specifically Dr R. Marty for his help in the synthesis of PEGDM. The support of D. Casari, L. Léoty and A. Trömel and the fruitful discussions with Prof. V. Michaud and E. Farmand-ashtiani are highly appreciated.

\section{References}

[1] A.S. Hoffman, Hydrogels for biomedical applications, Adv. Drug Deliv. Rev. 64 (2012) 18-23.

[2] R.E. Webber, C. Creton, H.R. Brown, J.P. Gong, Large strain hysteresis and mullins effect of tough double-network hydrogels, Macromolecules 40 (8) (2007) 2919-2927.

[3] M. Browning, T. Wilems, M. Hahn, E. Cosgriff-Hernandez, Compositional control of poly (ethylene glycol) hydrogel modulus independent of mesh size, 
J. Biomed. Mater. Res. Part A 98 (2) (2011) 268-273.

[4] J. Li, W.R. Illeperuma, Z. Suo, J.J. Vlassak, Hybrid hydrogels with extremely high stiffness and toughness, ACS Macro Lett. 3 (6) (2014) 520-523.

[5] G. Lake, A. Thomas, The strength of highly elastic materials, Proc. R. Soc. Lond. Ser. A Math. Phys. Sci. 300 (1460) (1967) 108-119.

[6] J.P. Maranchi, M.M. Trexler, Q. Guo, J.H. Elisseeff, Fibre-reinforced hydrogels with high optical transparency, Int. Mater. Rev. 59 (5) (2014) 264-296.

[7] T.D. Sargeant, A.P. Desai, S. Banerjee, A. Agawu, J.B. Stopek, An in situ forming collagen-PEG hydrogel for tissue regeneration, Acta Biomater. 8 (1) (2012) $124-132$.

[8] L.C. Lopérgolo, A.B. Lugão, L.H. Catalaini, Development of a poly(N-vinyl-2pyrrolidone)/poly (ethylene glycol) hydrogel membrane reinforced with methyl methacrylate-grafted polypropylene fibers for possible use as wound dressing, J. Appl. Polym. Sci. 86 (3) (2002) 662-666.

[9] D. Kai, M.P. Prabhakaran, B. Stahl, M. Eblenkamp, E. Wintermantel S. Ramakrishna, Mechanical properties and in vitro behavior of nanofiber-hydrogel composites for tissue engineering applications, Nanotechnology 23 (9) (2012) 095705.

[10] M. Feldmann, A.K. Bledzki, Bio-based polyamides reinforced with cellulosic fibres-processing and properties, Compos. Sci. Technol 100 (2014) 113-120.

[11] K.-Y. Lee, Y. Aitomäki, L.A. Berglund, K. Oksman, A. Bismarck, On the use of nanocellulose as reinforcement in polymer matrix composites, Compos. Sci. Technol. 105 (2014) 15-27.

[12] C. Chang, L. Zhang, Cellulose-based hydrogels: present status and application prospects, Carbohydr. Polym. 84 (1) (2011) 40-53.

[13] C. Eyholzer, A. Borges de Couraca, F. Duc, P.E. Bourban, P. Tingaut,
T. Zimmermann, et al., Biocomposite hydrogels with carboxymethylated, nanofibrillated cellulose powder for replacement of the nucleus pulposus, Biomacromolecules 12 (5) (2011) 1419-1427.

[14] S.S. Nair, J. Zhu, Y. Deng, A.J. Ragauskas, Hydrogels prepared from cross-linked nanofibrillated cellulose, ACS Sustain. Chem. Eng. 2 (4) (2014) 772-780.

[15] C.-C. Lin, K.S. Anseth, PEG hydrogels for the controlled release of biomolecules in regenerative medicine, Pharm. Res. 26 (3) (2009) 631-643.

[16] S. Lin-Gibson, S. Bencherif, J.A. Cooper, S.J. Wetzel, J.M. Antonucci, B.M. Vogel, et al., Synthesis and characterization of peg dimethacrylates and their hydrogels, Biomacromolecules 5 (4) (2004) 1280-1287.

[17] S. Josset, P. Orsolini, G. Siqueira, A. Tejado, P. Tingaut, T. Zimmermann, Energy consumption of the nanofibrillation of bleached pulp, wheat straw and recycled newspaper through a grinding process, Nord. Pulp Paper Res. J. 29 (2014) 167-175

[18] A.M. Kloxin, C.J. Kloxin, C.N. Bowman, K.S. Anseth, Mechanical properties of cellularly responsive hydrogels and their experimental determination, Adv. Mater. 22 (31) (2010) 3484-3494.

[19] M.-P. Lowys, J. Desbrieres, M. Rinaudo, Rheological characterization of cellulosic microfibril suspensions. Role of polymeric additives, Food Hydrocoll. 15 (1) (2001) 25-32.

[20] A.M. Schmocker, A. Khoushabi, B. Gantenbein-Ritter, S. Chan, H.M. Bonél, P.E. Bourban, et al., Minimally invasive photopolymerization in intervertebral disc tissue cavities, SPIE BiOS Int. Soc. Opt. Photonics (2014) 895206-895208.

[21] G.D. Nicodemus, S.J. Bryant, Cell encapsulation in biodegradable hydrogels for tissue engineering applications, Tissue Eng. Part B Rev. 14 (2) (2008) 149-165. 